\title{
Vorwort zur 2. Auflage
}

Die 2. Auflage wurde in großen Teilen komplett überarbeitet. Auch die Themenanordnung wurde teilweise verändert, um eine noch kohärentere Darstellung des Stoffs zu erreichen. Einige Themen sind in der neuen Auflage ausführlicher behandelt, wie z. B. die Quantentheorie des starren Körpers, die in vielen Bereichen, insbesondere in der Atom- und Kernphysik, benötigt wird. Die Wärmestrahlung, die in der ersten Auflage im einleitenden Kapitel über historische Experimente zur Entstehung der Quantenmechanik besprochen wurde, ist jetzt in das Kapitel Quantenstatistik eingearbeitet. Neu aufgenommen wurden die Vielteilchen-Green'schen Funktionen und deren erzeugendes Funktional sowie die zeitabhängige Hartree-Fock-Theorie.

Das Layout wurde komplett überarbeitet und an die neuen Vorgaben des Verlags angepasst. Wichtige Gleichungen sind eingerahmt, wichtige Aussagen farbig hinterlegt und bei besonderer Bedeutung zusätzlich mit dem Icon $\boldsymbol{\xi}$ versehen. Beweise sind durch ein $\boldsymbol{Z}$, Kommentare durch ein $\mathbf{i}$ gekennzeichnet.

Tübingen, im Juni 2019 Hugo Reinhardt 